PROCEEDINGS OF THE AMERICAN MATHEMATICAL SOCIETY

Volume 124, Number 7, July 1996

\title{
$p$-SEQUENTIALITY AND $p$-FRÉCHET-URYSOHN PROPERTY OF FRANKLIN COMPACT SPACES
}

\author{
S. GARCIA-FERREIRA AND V. I. MALYKHIN
}

(Communicated by Franklin D. Tall)

\begin{abstract}
Franklin compact spaces defined by maximal almost disjoint families of subsets of $\omega$ are considered from the view of its $p$-sequentiality and $p$-Fréchet-Urysohn-property for ultrafilters $p \in \omega^{*}$. Our principal results are the following: $\mathrm{CH}$ implies that for every $P$-point $p \in \omega^{*}$ there are a Franklin compact $p$-Fréchet-Urysohn space and a Franklin compact space which is not $p$-Fréchet-Urysohn; and, assuming $\mathrm{CH}$, for every Franklin compact space there is a $P$-point $q \in \omega^{*}$ such that it is not $q$-Fréchet-Urysohn. Some new problems are raised.
\end{abstract}

\section{INTRODUCTION}

Let us recall some definitions. All spaces are assumed to be completely regular and Hausdorff. The Greek letter $\omega$ will denote the first infinite ordinal. The set of all infinite subsets of $\omega$ is denoted by $[\omega]^{\omega}$. The Stone-Čech compactification of $\omega$ with the discrete topology is denoted by $\beta(\omega)$, which can be viewed as the set of all ultrafilters on $\omega$, where $\widehat{A}=\operatorname{cl}_{\beta(\omega)}(A)=\{q \in \beta(\omega): A \in q\}$, for $A \subseteq \omega$, is a basic clopen subset of $\beta(\omega)$. The remainder $\omega^{*}=\beta(\omega) \backslash \omega$ is the set of all free ultrafilters on $\omega$. For $A \in[\omega]^{\omega}$, let $A^{*}$ stand for $\widehat{A} \backslash A$. If $f: X \rightarrow Y$ is a continuous function, then $\bar{f}: \beta(X) \rightarrow \beta(Y)$ stands for the Stone-Cech extension of $f$. The Rudin-Keisler (pre-)order on $\omega^{*}$ is defined by $q \leq_{\mathrm{RK}} p$ if there is a function $f: \omega \rightarrow \omega$ such that $\bar{f}(p)=q$, for $p, q \in \omega^{*}$. The set of RK-predecessors of $p \in \omega^{*}$ is denoted by $P_{\mathrm{RK}}(p)=\left\{q \in \omega^{*}: q \leq_{\mathrm{RK}} p\right\}$. We say that $p$ is RK-equivalent to $q$ and write $p \approx_{\mathrm{RK}} q$, if $p \leq_{\mathrm{RK}} q$ and $q \leq_{\mathrm{RK}} p$. The Type of $p \in \omega^{*}$ is the set $T(p)=\left\{q \in \omega^{*}: p \approx_{\mathrm{RK}} q\right\}$ and it is not hard to see that $p \approx_{\mathrm{RK}} q$ iff there is a bijection $f: \omega \rightarrow \omega$ such that $\bar{f}(p)=q$. If $p \in \omega^{*}$, then $T(p)$ is a dense subset of $\omega^{*}$. A subset $N \subseteq \omega^{*}$ is said to be $R K$-dense in $\omega^{*}$ if for every $p \in \omega^{*}$ there is $q \in N$ such that $q \leq_{\text {RK }} p$. If $\Sigma \subseteq[\omega]^{\omega}$, then $\Sigma^{*}=\left\{A^{*}: A \in \Sigma\right\}$. An almost disjoint (AD) family of subsets of $\omega$ is a family $\Sigma$ of $[\omega]^{\omega}$ such that if $A, B \in \Sigma$ and $A \neq B$, then $|A \cap B|<\omega$ or $A^{*} \cap B^{*}=\varnothing$. If $\Sigma$ is not a proper subset of any AD family, then $\Sigma$ is called a maximal almost disjoint (MAD) family.

Our fundamental concept is the following.

Received by the editors July 5, 1993 and, in revised form, January 27, 1995.

1991 Mathematics Subject Classification. Primary 54A20, 54A35.

Key words and phrases. Ultrafilter, MAD family, Franklin compact space, Rudin-Keisler order, $p$-sequential, $p$-Fréchet Urysohn, ultra-sequential, ultra-Fréchet-Urysohn.

(C)1996 American Mathematical Society 
1.1. Definition (Katětov [Ka]). Let $\mathcal{F}$ be a filter on $\omega$. A point $x$ of a topological space $X$ is a $\mathcal{F}$-limit point of the sequence $\left(x_{n}\right)_{n<\omega}$ in $X$, we write $x=\mathcal{F}$-lim $x_{n}$ if $\left\{n<\omega: x_{n} \in V\right\} \in \mathcal{F}$ for every neighborhood $V$ of $x$.

Let us note that if $M$ is a nonempty closed subset of $\omega^{*}$ and $\mathcal{F}_{M}=\left\{A \in[\omega]^{\omega}\right.$ : $\left.M \subseteq A^{*}\right\}$, then $M=\left\{p \in \omega^{*}: \mathcal{F}_{M} \subseteq p\right\}=\bigcap\left\{A^{*}: A \in \mathcal{F}_{M}\right\}$ and $\mathcal{F}_{M}$ is a free filter on $\omega$. Conversely, if $\mathcal{F}$ is a free filter on $\omega$ and $M_{\mathcal{F}}=\bigcap\left\{A^{*}: A \in \mathcal{F}\right\}$, then $\mathcal{F}=\left\{A \in[\omega]^{\omega}: M_{\mathcal{F}} \subseteq A^{*}\right\}, M_{\mathcal{F}}=\left\{p \in \omega^{*}: \mathcal{F} \subseteq p\right\}$ and $M_{\mathcal{F}}$ is a nonempty closed subset of $\omega^{*}$. Hence, we have that $M=M_{\mathcal{F}_{M}}$ and $\mathcal{F}=\mathcal{F}_{M_{\mathcal{F}}}$ for every nonempty closed subset $M$ of $\omega^{*}$ and for every free filter $\mathcal{F}$ on $\omega$. Thus, throughout this paper, we will not distinguish between nonempty closed subsets of $\omega^{*}$ and the corresponding free filters on $\omega$. This correspondence allows us to see that $\mathcal{F}$-limits, for an arbitrary free filter $\mathcal{F}$ on $\omega$, may be defined in terms of $p$-limits for $p \in \omega^{*}$ as is stated in the next lemma.

1.2. Lemma. Let $\mathcal{F}$ be a free filter on $\omega$, and let $\left(x_{n}\right)_{n<\omega}$ be a sequence in a space $X$. Then, $x=\mathcal{F}$ - $\lim x_{n}$ iff $x=p$-lim $x_{n}$ for all $p \in M_{\mathcal{F}}$.

Proof. Only the sufficiency requires proof. Assume that there is a neighborhood $V$ of $x$ such that $A=\left\{n<\omega: x_{n} \in V\right\} \notin \mathcal{F}$. Then, $M_{\mathcal{F}}$ is not contained in $A^{*}$. Hence, we pick $p \in M_{\mathcal{F}} \backslash A^{*}$. But, we have that $\left\{n<\omega: x_{n} \in V\right\} \in p$, since $x=p$-lim $x_{n}$, which is a contradiction.

By using the $\mathcal{F}$-limits we may generalize sequentiality and Fréchet-Urysohnproperty as follows:

1.3. Definition. Let $\mathcal{F}$ be a free filter on $\omega$ and $X$ a space. Then

(1) (Kombarov [Ko]) $X$ is $\mathcal{F}$-sequential if for every nonclosed subset $A$ of $X$ there are a sequence $\left(x_{n}\right)_{n<\omega}$ in $A$ and $x \in X \backslash A$ such that $x=\mathcal{F}$ - $\lim x_{n}$;

(2) (Comfort-Ponomarov-Savchenko) $X$ is a $\mathrm{FU}(\mathcal{F})$-space if for each $A \subseteq X$ and each $x \in \operatorname{cl}(A)$ there is a sequence $\left(x_{n}\right)_{n<\omega}$ in $A$ such that $x=\mathcal{F}$ - $\lim x_{n}$;

(3) (Malykhin) $X$ is ultra-sequential (resp., ultra-FU) if $X$ is $p$-sequential (resp., $\mathrm{FU}(p)$-space) for every $p \in \omega^{*}$.

The next lemma is a direct consequence of Lemma 1.2.

1.4. Lemma. Let $\mathcal{F}$ be a free filter on $\omega$ and $X$ a space. Then

(1) $X$ is $\mathcal{F}$-sequential iff for every nonclosed subset $A$ of $X$ there are a sequence $\left(x_{n}\right)_{n<\omega}$ and $x \in X \backslash A$ such that $x=p$-lim $x_{n}$ for all $p \in M_{\mathcal{F}}$;

(2) $X$ is a $\mathrm{FU}(\mathcal{F})$-space iff for each $A \subseteq X$ and each $x \in \operatorname{cl}(A)$ there is a sequence $\left(x_{n}\right)_{n<\omega}$ in $A$ such that $x=p-\lim x_{n}$ for all $p \in M_{\mathcal{F}}$.

Notice from 1.4 that if $X$ is $\mathcal{F}$-sequential (resp., a $\mathrm{FU}(\mathcal{F})$-space) for a free filter $\mathcal{F}$ on $\omega$, then $X$ is $p$-sequential (resp., a $\mathrm{FU}(p)$-space) for all $p \in M_{\mathcal{F}}$. The converse does not hold in general; for instance, if $q<_{\mathrm{RK}} p$, then the subspace $\xi(q)=\omega \cup\{q\}$ of $\beta(\omega)$ is both a $\mathrm{FU}(q)$-space and a $\mathrm{FU}(p)$-space, but it is not a $\mathrm{FU}\left(\mathcal{F}_{M}\right)$-space, where $M=\{p, q\}$. If $\mathcal{F}$ is a free filter on $\omega$, then $\mathcal{F}$-sequentiality and $\mathrm{FU}(\mathcal{F})$-property coincide with the concepts of strong $M_{\mathcal{F}}$-sequentiality (see $[\mathrm{Ko}]$ ) and $\mathrm{SFU}\left(M_{\mathcal{F}}\right)$-property (see $[\mathrm{GT}],[\mathrm{Koc}]$ ), respectively. If $\mathcal{F}_{r}$ is the Fréchet filter on $\omega$ (i.e., the filter generated by the cofinite subsets of $\omega$ ), then sequentiality and Fréchet-Urysohn-property agree with $\mathcal{F}_{r}$-sequentiality and $\mathrm{FU}\left(\mathcal{F}_{r}\right)$-property, respectively. More general, if $\operatorname{int}_{\omega^{*}}\left(M_{\mathcal{F}}\right) \neq \varnothing$ for a free filter $\mathcal{F}$ on $\omega$, then sequentiality $=\mathcal{F}$-sequentiality and Fréchet-Urysohn-property $=\mathrm{FU}(\mathcal{F})$-property. For a nonempty closed subset $M$ of $\omega^{*}, \mathrm{FU}(M)$-space will mean $\mathrm{FU}\left(\mathcal{F}_{M}\right)$-space. 
The basic relationship between Rudin-Keisler order and the concepts given in Definition 1.3 is established in the following lemma.

1.5. Lemma (Ferreira $[\mathrm{G}]$ ). For $p, q \in \omega^{*}$, the following are equivalent.

(1) $q \leq \mathrm{RK} p$

(2) every $q$-sequential space is p-sequential;

(3) every $\mathrm{FU}(q)$-space is a $\mathrm{FU}(p)$-space.

For $p \in \omega^{*}$ and a subset $Y$ of a space $X$, we define $Y^{p}=\left\{x \in X: x=p\right.$ - $\lim x_{n}$ for some sequence $\left(x_{n}\right)_{n<\omega}$ in $Y$ \}. By induction, we define $Y_{0}^{p}=Y$ and, for every ordinal number $\nu, Y_{\nu}^{p}=\left(Y_{\mu}^{p}\right)^{p}$ if $\nu=\mu+1$ and $Y_{\nu}^{p}=\bigcup_{\lambda<\nu} Y_{\lambda}^{p}$ if $\nu$ is a limit ordinal. Notice that if $X$ is a $p$-sequential space, then $\operatorname{cl}_{X}(Y)=Y_{\omega_{1}}^{p}=\bigcup_{\nu<\omega_{1}} Y_{\nu}^{p}$ for each $Y \subseteq X$. Hence, we have that a space $X$ is $p$-sequential iff the ordinal number

$$
\sigma_{p}(X)=\min \left\{\nu \leq \omega_{1}: \operatorname{cl}_{X}(Y)=Y_{\nu}^{p} \text { for all } Y \subseteq X\right\}
$$

exists. If $X$ is $p$-sequential, then $\sigma_{p}(X)$ is called the degree of $p$-sequentiality of $X$. Observe that a space $X$ is a $\mathrm{FU}(p)$-space iff $\sigma_{p}(X)=1$.

\section{FRANKLIN COMPACT SPACES}

We start with the definition of Franklin compact space: For a MAD family $\Sigma$, the Franklin compact space $\mathcal{F}(\Sigma)$ of $\Sigma$ is defined as follows: $\mathcal{F}(\Sigma)$ is a factor-space of $\beta(\omega)$ in which every $A^{*}$, for $A \in \Sigma$, is identified with a single point and $\omega^{*} \backslash \bigcup \Sigma^{*}$ is also identified with a single point $\infty$; hence, the point set of $\mathcal{F}(\Sigma)$ is $\omega \cup\left\{\left\{A^{*}\right\}: A \in\right.$ $\Sigma\} \cup\{\infty\}$. Franklin compact spaces were introduced in $[\mathrm{F}]$, and they have been studied in many papers, for instance, $\left[\mathrm{B}_{1}\right],\left[\mathrm{B}_{2}\right],\left[\mathrm{Ma}_{1}\right]$ and $\left[\mathrm{Ma}_{2}\right]$. It is known that Franklin compact spaces are sequential spaces with degree of sequentiality equal to 2 and are not Fréchet-Urysohn. In this section, we will consider these spaces from the view of $p$-sequentiality and $\mathrm{FU}(p)$-property for $p \in \omega^{*}$. So $\mathcal{F}(\Sigma)$ is ultrasequential and its degree of $p$-sequentiality is not greater than 2 , for every $p \in \omega^{*}$. The next two lemmas are fundamental in the study of the $p$-sequentiality and the $\mathrm{FU}(p)$-property of Franklin compact spaces.

2.1. Lemma. Let $p \in \omega^{*}$ and $\Sigma$ a MAD family. Then, $\mathcal{F}(\Sigma)$ is not a $\mathrm{FU}(p)$-space if and only if there is $C \in[\omega]^{\omega}$ such that

$$
C^{*} \backslash \bigcup \Sigma^{*} \neq \varnothing \quad \text { and } \quad P_{\mathrm{RK}}(p) \cap C^{*} \subseteq \bigcup \Sigma^{*} .
$$

Proof. Necessity: If $\sigma_{p}(\mathcal{F}(\Sigma))=2$, then there must be $C \in[\omega]^{\omega}$ such that $\infty \in$ $\operatorname{cl}(C)$ and no sequence of $C p$-converges to $\infty$. Let $q \in P_{\mathrm{RK}}(p) \cap C^{*}$. Then, we may find $f: \omega \rightarrow C$ such that $\bar{f}(p)=q$. Since $(f(n))_{n<\omega}$ is a sequence in $C$ and $q=p$-lim $f(n)$, we have that $q \in A^{*}$ for some $A \in \Sigma$. This shows that $P_{\mathrm{RK}}(p) \cap C^{*} \subseteq \bigcup \Sigma^{*}$.

Sufficiency: Let $C \in[\omega]^{\omega}$ satisfy that $C^{*} \backslash \bigcup \Sigma^{*} \neq \varnothing$ and $P_{\mathrm{RK}}(p) \cap C^{*} \subseteq \bigcup \Sigma^{*}$. It is enough to prove that no sequence of $C p$-converges to $\infty$ in $\mathcal{F}(\Sigma)$. Indeed, let $f: \omega \rightarrow C$ be such that $\bar{f}(p)=q \in \omega^{*}$. Then $q \in P_{\mathrm{RK}}(p) \cap C^{*} \subseteq \bigcup \Sigma^{*}$ and so there is $A \in \Sigma$ for which $q \in A^{*}$; hence, $A^{*}=p$ - $\lim f(n)$.

2.2. Lemma (Boldjiev and Malykhin $[\mathrm{BM}]$ ). Let $\Sigma$ be an AD family. If $p \in \omega^{*}$ is not a P-point, then $T(p)$ is not contained in $\bigcup \Sigma^{*}$.

From 2.2 it follows that if $T(p) \subseteq \bigcup \Sigma^{*}$ for some $\mathrm{AD}$ family $\Sigma$, then $p$ must be a $P$-point. The following theorem is proved in $[\mathrm{BM}]$ : now it can be considered as a consequence of Lemmas 2.1 and 2.2. 
2.3. Theorem (Boldjiev and Malykhin). Every Franklin compact space is a $\mathrm{FU}(p)$-space for each non-P-point $p \in \omega^{*}$.

2.4. Corollary (Boldjiev and Malykhin). If there are not P-points in $\omega^{*}$, then every Franklin compact space is ultra-FU.

The existence of $P$-points in $\omega^{*}$ is independent of the axioms of ZFC. In fact, W. Rudin $[\mathrm{R}]$ showed that $\mathrm{CH}$ implies the existence of $P$-points in $\omega^{*}$ and Shelah [W], [Mi] constructed a model of ZFC in which there are not $P$-points in $\omega^{*}$. Hence, in Shelah's model the Franklin compact spaces are ultra-FU. So, it is natural to ask whether every Franklin compact space is not a $\mathrm{FU}(p)$-space whenever $p \in \omega^{*}$ is a $P$-point. We shall show that $\mathrm{CH}$ implies that for every $P$-point $p \in \omega^{*}$ there is a MAD family $\Sigma$ such that $\mathcal{F}(\Sigma)$ is not a $\mathrm{FU}(p)$-space.

Let $\rho$ be the smallest cardinal, such that if $\mathcal{A}$ is a centered family of clopen subsets of $\omega^{*}$ and $|\mathcal{A}|<\mathfrak{c}$, then $\operatorname{int}(\bigcap \mathcal{A}) \neq \varnothing$. It is well known that $\rho=\mathfrak{c}$ under MA.

2.5. Theorem $(\rho=\mathfrak{c})$. If $N$ is a nowhere dense closed subset of $\omega^{*}$, then there is a MAD family $\Sigma$ such that $N \subseteq \omega^{*} \backslash \bigcup \Sigma^{*}$ and $\mathcal{F}(\Sigma)$ is a $\mathrm{FU}(N)$-space; hence, $\mathcal{F}(\Sigma)$ is a $\mathrm{FU}(p)$-space for all $p \in N$.

Proof. The desired family will be constructed by transfinite induction. Enumerate the set $\left\{C \in[\omega]^{\omega}: C^{*} \cap N=\varnothing\right\}$ as $\left\{C_{\alpha}: \alpha<\mathfrak{c}\right\}$. We define $A_{0}=C_{0}$ and $N_{0}=N$. Suppose that $A_{\beta} \in[\omega]^{\omega}$ and a nowhere dense closed subset $N_{\beta}$ have been defined for each $\beta<\alpha<\mathfrak{c}$, where $\alpha$ is a fixed ordinal smaller than $\mathfrak{c}$, so that

(1) $\Sigma_{\beta}=\left\{A_{\gamma}: \gamma<\beta\right\}$ is $\mathrm{AD}$ for each $\beta<\alpha$;

(2) $\left(\bigcup \Sigma_{\beta}^{*}\right) \cap\left(\bigcup_{\gamma<\beta} N_{\gamma}\right)=\varnothing$ for each $\beta<\alpha$;

(3) $N_{\beta} \subseteq \bigcup\left\{P_{\mathrm{RK}}(p): p \in N\right\}$ and $N_{\beta} \cap P_{\mathrm{RK}}(p) \neq \varnothing$ for each $\beta<\alpha$; and

(4) if $C_{\beta}^{*}$ is not contained in $\bigcup \Sigma_{\beta}^{*}$, then $A_{\beta}^{*} \subseteq C_{\beta}^{*} \backslash\left[\left(\bigcup \Sigma_{\beta}^{*}\right) \cup\left(\bigcup_{\gamma \leq \beta} N_{\gamma}\right)\right]$.

Consider $C_{\alpha}^{*}$ and $\Sigma_{\alpha}=\left\{A_{\beta}: \beta<\alpha\right\}$. If $C_{\alpha}^{*} \subseteq \bigcup \Sigma_{\alpha}^{*}$, then we let $N_{\alpha}=\varnothing$. If this is not the case, then put $W=\operatorname{int}\left(C_{\alpha}^{*} \backslash\left[\left(\bigcup \Sigma_{\alpha}^{*}\right) \cup\left(\bigcup_{\beta<\alpha} N_{\beta}\right)\right]\right)$. Since $\rho=\mathfrak{c}$, we have that $W \neq \varnothing$. Hence, we choose $B \in[\omega]^{\omega}$ so that $B^{*} \subseteq W$. Now, we fix a one-to-one function $f_{\alpha}: \omega \rightarrow B$ and set $N_{\alpha}=\bar{f}_{\alpha}(N)$. Then we choose $A_{\alpha} \in[\omega]^{\omega}$ such that $A_{\alpha}^{*} \subseteq B^{*} \backslash N_{\alpha}$. Thus, we have defined $A_{\alpha}$ and $N_{\alpha}$. It is not hard to verify that $\Sigma=\left\{A_{\alpha}: \alpha<\mathfrak{c}\right\}$ is the required MAD family.

It follows from 2.5, in particular, that if $\rho=\mathfrak{c}$ and $K \subseteq \omega^{*}$ satisfies $|K|<\mathfrak{c}$, then there is a MAD family $\Sigma$ such that $\mathcal{F}(\Sigma)$ is a $\mathrm{FU}(p)$-space for every $p \in \operatorname{cl}(K)$.

The next result, for only one $P$-point of $\omega^{*}$, is contained in [BM, Example 1], but there is a small defect in its proof there. Here, we give a more general statement and the correct proof.

2.6. Theorem $(\mathrm{CH})$. If $p_{\alpha} \in \omega^{*}$ is a P-point for every $\alpha<\mathfrak{c}$, then there exists a MAD family $\Sigma$ such that $\bigcup_{\alpha<\mathfrak{c}} P_{\mathrm{RK}}\left(p_{\alpha}\right) \subseteq \bigcup \Sigma^{*}$ and hence $\sigma_{p_{\alpha}}(\mathcal{F}(\Sigma))=2$ for each $\alpha<\mathfrak{c}$.

Proof. Let $p_{\alpha} \in \omega^{*}$ be a $P$-point for every $\alpha<\mathfrak{c}$. Notice that each element of $\bigcup_{\alpha<\mathfrak{c}} P_{\mathrm{RK}}\left(p_{\alpha}\right)$ is also a $P$-point of $\omega^{*}$. We may enumerate $\bigcup_{\alpha<\mathfrak{c}} P_{\mathrm{RK}}\left(p_{\alpha}\right)$ as $\left\{q_{\mu}: \mu<\omega_{1}\right\}$; this is possible because $\left|\bigcup_{\alpha<\mathfrak{c}} P_{\mathrm{RK}}\left(p_{\alpha}\right)\right|=2^{\omega}=\omega_{1}$. To define the MAD family we proceed by transfinite induction. Let $\left\{A_{n}: n<\omega\right\}$ be a partition of $\omega$ in infinite subsets. Suppose that $A_{\mu}$ has been defined for $\mu<\nu<\omega_{1}$ so that

(1) $\left\{A_{\mu}: \mu<\nu\right\}$ is an AD family; and 
(2) $\left\{q_{\mu}: \mu<\nu\right\} \subseteq \bigcup\left\{A_{\mu}^{*}: \mu<\nu\right\}$.

If $q_{\nu} \in \bigcup\left\{A_{\mu}^{*}: \mu<\nu\right\}$, then we choose any $A_{\nu} \in[\omega]^{\omega}$ such that $\left|A_{\nu} \cap A_{\mu}\right|<\omega$ for all $\mu<\nu$. Otherwise, we take $A_{\nu} \in q_{\nu}$ so that $A_{\nu}^{*} \cap A_{\mu}^{*}=\varnothing$ for every $\mu<\nu$. Set $\Sigma=\left\{A_{\mu}: \mu<\nu\right\}$. By construction, we have that $\bigcup_{\alpha<\mathfrak{c}} P_{\mathrm{RK}}\left(p_{\alpha}\right) \subseteq \bigcup\left\{A_{\mu}^{*}: \mu<\right.$ $\left.\omega_{1}\right\}$. Now, if $A \in[\omega]^{\omega}$, then there are $\mu, \nu<\omega_{1}$ such that $q_{\mu} \in A^{*} \cap A_{\nu}^{*}$ and so $A \cap A_{\nu}$ is infinite. This shows that $\Sigma$ is a MAD family. From 2.1 it follows that $\sigma_{p_{\alpha}}(\mathcal{F}(\Sigma))=2$ for each $\alpha<\mathfrak{c}$.

A solution to the following problem could provide some information about the $\mathrm{FU}(p)$-property of Franklin compact spaces for the case when $p \in \omega^{*}$ is a $P$-point.

2.7. Problem. Is it possible that an ultra-FU Franklin compact space exists if $P$-points of $\omega^{*}$ exist?

The proof of the next theorem is analogous to the previous one.

2.8. Theorem (MA). For every $P_{\mathfrak{c}}$-point $p \in \omega^{*}$ there exists a MAD family $\Sigma$ such that $P_{\mathrm{RK}}(p) \subseteq \bigcup \Sigma^{*}$ and hence $\sigma_{p}(\mathcal{F}(\Sigma))=2$.

2.9. Problem. Assume $[M A+\neg C H]$ and let $p \in \omega^{*}$ be a $P_{\kappa}$-point for some $\omega<\kappa<\mathfrak{c}$ (such $P_{\kappa}$-point exists under $[M A+\neg C H]$, see [S]).

Is there a MAD family $\Sigma$ family that:

(a) $T(p) \subseteq \bigcup \Sigma^{*}$ or

(b) $P_{\mathrm{RK}}(p) \subseteq \bigcup \Sigma^{*}$ ?

We turn now to prove that $\mathrm{CH}$ implies that for every MAD family $\Sigma$ there is a $P$-point $q \in \omega^{*}$ such that $\mathcal{F}(\Sigma)$ is not a FU $(q)$-space. We need the next lemma.

2.10. Lemma. Let $\Sigma$ be a MAD family. If $f: \omega \rightarrow \omega$ is finite-to-one and onto, then $f^{-1}[\Sigma]=\left\{f^{-1}(A): A \in \Sigma\right\}$ is also a MAD family.

Proof. Let $T=f^{-1}[\Sigma]$. Since $\Sigma$ is $\mathrm{AD}$ and $f$ is finite-to-one, we have that $\mid f^{-1}(A) \cap$ $f^{-1}(B) \mid<\omega$ whenever $A, B \in \Sigma$ and $A \neq B$. Now, let $B \in[\omega]^{\omega}$. Since $f[B] \in[\omega]^{\omega}$, there is $A \in \Sigma$ such that $|A \cap f[B]|=\omega$ and so $\left|f^{-1}(A) \cap B\right|=\omega$. Thus, $T$ is a MAD family.

2.11. Theorem $(\mathrm{CH})$. For every $M A D$ family $\Sigma$ there is a P-point $p \in \omega^{*}$ such that $P_{\mathrm{RK}}(p) \subseteq \bigcup \Sigma^{*}$ and hence $\sigma_{p}(\mathcal{F}(\Sigma))=2$.

Proof. Let $\mathcal{F}=\{f \mid f: \omega \rightarrow \omega$ is finite-to-one and onto $\}$ and $\Sigma=\left\{A_{\alpha}: \alpha<\omega_{1}\right\}$. Enumerate $\mathcal{F}$ as $\left\{f_{\alpha}: \alpha<\omega_{1}\right\}$. We proceed by transfinite induction. Set $B_{0}=A_{0}$, and assume that $B_{\beta}$ and $A_{\gamma_{\beta}} \in \Sigma$ have been defined for each $\beta<\alpha$ so that

(1) $B_{\beta}^{*} \subseteq\left[B_{0} \cap f_{\beta}^{-1}\left(A_{\gamma_{\beta}}\right)\right]^{*}$ for $\beta<\alpha$; and

(2) $\left\{B_{\beta}: \beta<\alpha\right\}$ has the finite intersection property. Since $\left\{B_{\beta}: \beta<\alpha\right\}$ has the finite intersection property, there is $D \in[\omega]^{\omega}$ for which $D^{*} \subseteq \bigcap_{\beta<\alpha} B_{\beta}^{*}$. By Lemma 2.10, we may find $A_{\gamma_{\alpha}} \in \Sigma$ such that $\left|D \cap f_{\alpha}^{-1}\left(A_{\gamma_{\alpha}}\right)\right|=\omega$. Then, we put $B_{\alpha}=D \cap f_{\alpha}^{-1}\left(A_{\gamma_{\alpha}}\right)$. Thus, $\left\{B_{\alpha}: \alpha<\omega_{1}\right\}$ has the finite intersection property and hence there is $p \in \omega^{*}$ such that $\left\{B_{\alpha}: \alpha<\omega_{1}\right\} \subseteq p$. So $p \in A_{0}^{*}$. If $f: \omega \rightarrow \omega$ is a bijection, then $f=f_{\alpha}$ for some $\alpha<\omega_{1}$; hence, $\bar{f}(p) \in A_{\gamma_{\alpha}}^{*}$. This shows that $T(p) \subseteq \bigcup \Sigma^{*}$. According to 2.2 , we have that $p$ must be a $P$-point of $\omega^{*}$. Notice, in general, that if $q \leq_{\mathrm{RK}} p$ and $p \in \omega^{*}$ is a $P$-point, then there is a finiteto-one surjection $f: \omega \rightarrow \omega$ such that $\bar{f}(p)=q$. We claim that $P_{\mathrm{RK}}(p) \subseteq \bigcup \Sigma^{*}$. Indeed, fix $q \in P_{\mathrm{RK}}(p)$. Then, there is $\alpha<\omega_{1}$ such that $\bar{f}_{\alpha}(p)=q$. Since $p \in\left[f_{\alpha}^{-1}\left(A_{\gamma_{\alpha}}\right)\right]^{*}, q=\bar{f}_{\alpha}(p) \in A_{\gamma_{\alpha}}^{*}$. This proves the claim. 
In a similar way we may prove the following.

2.12. Theorem (MA). For every MAD family $\Sigma$ there is a $P_{\mathfrak{c}}$-point $p \in \omega^{*}$ such that $P_{\mathrm{RK}}(p) \subseteq \bigcup \Sigma^{*}$ and hence $\sigma_{p}(\mathcal{F}(\Sigma))=2$.

We could predict something like Theorems 2.14 and 2.15 after knowing Malykhin's result $\left[\mathrm{Ma}_{3}\right.$, Theorem 1], about the coincidence of ultra-FU and ultrasequentiality with Fréchet-Urysohn property and sequentiality, respectively, under the assumption $n\left(\omega^{*}\right)>\mathfrak{c}$, where $n(X)$ is the Novak number of $X$ (i.e., the smallest power of a family of nowhere dense subsets of $X$ covering $X)$. Indeed, under $\mathrm{CH}$, MA or $n\left(\omega^{*}\right)>\mathfrak{c}$ a Franklin compact space $\mathcal{F}(\Sigma)$ cannot be ultra-FU (i.e., there is $p \in \omega^{*}$ such that $\left.\sigma_{p}(\mathcal{F}(\Sigma))>1\right)$ since it is not Fréchet-Urysohn. As a consequence of this result and Lemma 1.5 we have the following two theorems.

2.13. Problem. Under $\mathrm{CH}$, is it true that for every MAD family $\Sigma$ there is a $P$-point $p \in \omega^{*}$ such that $\mathcal{F}(\Sigma)$ is a $\mathrm{FU}(p)$-space?

2.14. Theorem. Let $\Sigma$ be a MAD family. Then, $\mathcal{F}(\Sigma)$ is ultra-FU if and only if $\left\{p \in \omega^{*}: \mathcal{F}(\Sigma)\right.$ is a $\mathrm{FU}(p)$-space $\}$ is RK-dense in $\omega^{*}$.

The next result is a direct application of Malykhin's Theorem quoted above and 2.14 .

2.15. Theorem $\left(n\left(\omega^{*}\right)>\mathfrak{c}\right)$. Let $\Sigma$ be a MAD family. Then, the set $\left\{p \in \omega^{*}\right.$ : $\mathcal{F}(\Sigma)$ is a $\mathrm{FU}(p)$-space $\}$ is not $\mathrm{RK}$-dense in $\omega^{*}$.

We formulate and prove the following proposition that is stronger than Malykhin's theorem.

2.16. Proposition $\left(n\left(\omega^{*}\right)>\mathfrak{c}\right)$. Let $\mathfrak{C}$ be a family of nonempty closed subsets of $\omega^{*}$ such that $|\mathfrak{C}| \leq \mathfrak{c}$ and $\bigcup \mathfrak{C}$ is RK-dense in $\omega^{*}$. Then, $\operatorname{int}(F) \neq \varnothing$ for some $F \in \mathfrak{C}$.

Proof. We have that for every $p \in \omega^{*}$ there are $F_{p} \in \mathfrak{C}$ and a function $f_{p}: \omega \rightarrow \omega$ such that $p \in \bar{f}_{p}^{-1}\left(F_{p}\right)$. Hence, $\left\{\bar{f}_{p}^{-1}\left(F_{p}\right): p \in \omega^{*}\right\}$ is a family of nonempty closed subsets of $\omega^{*}$ and covers $\omega^{*}$. But this family has power less than or equal to $\mathfrak{c}$. So there must be $F \in \mathfrak{C}$ such that $\operatorname{int}(F) \neq \varnothing$.

We proceed to prove, under the assumption of $n\left(\omega^{*}\right)>\mathfrak{c}$, a result more general than the one given in Theorem 2.11: it is an immediate consequence of 2.1 and 2.16 .

2.17. Theorem $\left(n\left(\omega^{*}\right)>\mathfrak{c}\right)$. Let $\Sigma_{\alpha}$ be a MAD family for every $\alpha<\mathfrak{c}$. Then, there is (a P-point) $p \in \omega^{*}$ such that $\mathcal{F}\left(\Sigma_{\alpha}\right)$ is not a $\mathrm{FU}(p)$-space for all $\alpha<\mathfrak{c}$.

Proof. For each $\alpha<\mathfrak{c}$, put $F_{\alpha}=\omega^{*} \backslash \bigcup \Sigma_{\alpha}^{*}$. Consider the set $\mathfrak{C}=\left\{F_{\alpha}: \alpha<\mathfrak{c}\right\}$. Since $\operatorname{int}\left(F_{\alpha}\right)=\varnothing_{\alpha}$ for each $\alpha<\mathfrak{c}$, by 2.16 , there is $p \in \omega^{*}$ such that $P_{\mathrm{RK}}(p) \cap \bigcup \mathfrak{C}=$ $\varnothing$ and so $P_{\mathrm{RK}}(p) \subseteq \omega^{*} \backslash \bigcup \mathfrak{C}=\bigcap_{\alpha<\mathrm{c}}\left[\bigcup \Sigma_{\alpha}^{*}\right]$. Lemma 2.1 implies that $\mathcal{F}\left(\Sigma_{\alpha}\right)$ is not a $\mathrm{FU}(p)$-space for all $\alpha<\mathfrak{c}$.

The next problem now appears to be natural.

2.18. Problem. Is it possible that some $p \in \omega^{*}$ exists such that every Franklin compact space is not a $\mathrm{FU}(p)$-space? 
Perhaps it will be so in models obtained from GCH and by adding greater than $\omega_{1}$ of new Cohen reals.

We recall that, for $p \in \omega^{*}$, a space $X$ is a strictly $\mathrm{FU}(p)$-space if $x \in \bigcap_{n<\omega} \operatorname{cl}_{X}\left(Y_{n}\right)$, where $Y_{n} \subseteq X$ for each $n<\omega$; then for each $n<\omega$ there is $x_{n} \in Y_{n}$ such that $x=p$-lim $x_{n}$.

2.19. Problem. Let $p \in \omega^{*}$, and let $\Sigma$ be a MAD family. Can $\mathcal{F}(\Sigma)$ be a strictly $\mathrm{FU}(p)$-space?

\section{REFERENCES}

[B $\left.\mathrm{B}_{1}\right]$ A. I. Baškirov, On the classification of quotient mappings and compact sequential spaces, Soviet Math. Dokl. 15 (1974), 1104-1109. MR 50:11159

$\left[\mathrm{B}_{2}\right] \quad$ On maximal disjoint systems and Franklin bicompacta, Soviet Math. Dokl. 19 (1978), 864-868. MR 80m:54032

$[\mathrm{BM}]$ B. Boldjiev and V. I. Malichin, The sequentiality is equivalent to the $\mathcal{F}$-Fréchet-Urysohn property, Comment. Math. Univ. Carolin. 31 (1990), 23-25. MR 91g:54005

[F] S. P. Franklin, Spaces in which sequences suffice II, Fund. Math. 61 (1967), 51-56. MR 36:5882

[G] S. Garcia-Ferreira, On FU $(p)$-spaces and p-sequential spaces, Comment. Math. Univ. Carolin. 32 (1991), 161-171. MR 92m:54006

[GT] S. Garcia-Ferreira and A. Tamariz-Mascarua, On p-sequential p-compact spaces, Comment. Math. Univ. Carolin. 34 (1993), 347-356. MR 94m:54008

[Ka] M. Katětov, Products of filters, Comment. Math. Univ. Carolin. 9 (1968), 173-189. MR 40:3496

[Koč] L. D. Kočinac, A generalization of chain-net spaces, Publ. Inst. Math. (Beograd) 44 (58) (1988), 109-114. MR 90h:54006

[Ko] A. P. Kombarov, On the theorem of A. M. Stone, Soviet Math. Dokl. 27 (1983), 544-547. MR 85j:54029

[Ma $]$ V. I. Malykhin, Sequential bicompacta: Čech-Stone extensions and $\pi$-points, Moscow Univ. Math. Bull. 30 (1975), 18-23. MR 51:11432

$\left[\mathrm{Ma}_{2}\right] \ldots$, On sequential and Fréchet-Urysohn bicompacta, Moscow Univ. Math. Bull. 31 (1976) 33-37. MR 58:7534

[Ma3 $]$, The sequentiality and the Fréchet-Urysohn property with respect to ultrafilters, Acta Univ. Carolin.-Math. Phys. 31 (1990), 65-69. MR 92h:54006

[Mi] Ch. Mills, An easier proof of the Shelah P-point independence theorem (to appear).

[R] W. Rudin, Homogeneity problems in the theory of Čech compactifications, Duke Math. J. 23 (1956), 409-419. MR 18:324

[S] A. Szymanski, The existence of $P(\alpha)$-points of $N^{*}$ for $\aleph_{0}<\alpha<\mathfrak{c}$, Colloq. Math. 37 (1977), 279-184. MR 58:7571

[W] E. L. Wimmers, The Shelah P-point independence theorem, Israel J. Math. 43 (1982), 28-48. MR 85e:03118

Instituto de Matematicas, Unidad Morelia (UNAM), Nicolás Romero 150, Morelia, MiCHOACAN 58000, MÉXICO

E-mail address: garcia@servidor.unam.mx

E-mail address: sgarcia@zeus.ccu.umich.mx

State Academy of Management, Ruazanskij Prospekt 99, Moscow, Russia 109542

E-mail address: matem@acman.msk.su 